\title{
LE LAC DE CONSTANCE
}

\author{
par Jacques de MOUSTIER \\ Ingénieur des Eaux et Forêts, Commissaire français en Allemagne \\ pour la pêche du Lac de Constance et des eaux limitrophes du bassin du Rhin.
}

\section{L'EXPLOITATION PISGIGOLE DU LAG}

\section{I. - ENGINS ET MODES DE PECHE}

Les engins et modes de pêche sont extrêmement variés suivant l'espèce de poisson que l'on recherche, les lieux, époques et heures de la pêche.

Comme partout ailleurs, les engins susceptibles de blesser le poisson ou de l'étourdir (harpons, armes à feu, etc...) ainsi que les poisons et explosifs sont prohibés. Les engins autorisés se divisent en trois groupes :

-_ Les lignes;

- Les filets;

- Les verveux.

\section{1. - Les lignes.}

Parmi celles-ci on distingue :

- La ligne ò main, avec ou sans gaule, dont l'appât est naturel (vers, larves, pain, etc...) ou artificiel (mouches, devons, etc...). Elle est utilisée de la rive. Son emploi, en Würtemberg et en Suisse, n'entraîne le paiement d'aucune taxe.

- La ligne d̀ secousses ou " à la touche " (Zockangel ou Zockschnur). Ligne en soie ou crin de cheval de 20 à 40 mètres de longueur tendue par un plomb, munie latéralement d'hameçons simples ou multiples montés sur empiles de 20 centimètres environ, et appâtés avec des poissons vivants ou morts, des vers ou des larves d'éphémères. Elle permet surtout la capture de la Perche, mais aussi du Brochet et parfois de Corégones à gros yeux et Féras. Elle est utilisée plus particulièrement dans le Lac Inférieur et en hiver, à bord d'un bateau se mouvant lentement ou au repos.

- La ligne trainante (Schleppangel ou Schwemmschnur), ligne de 50 à 100 mètres, garnie de plombs d'enfoncement choisis de telle sorte 
que la ligne se maintienne dans la zone où se trouve le poisson. Elle est tirée à l'aide d'un treuil, à partir d'un bateau en mouvement et elle est munie d'appâts naturels ou artificiels. Elle est appréciée surtout par les pêcheurs sportifs qui en manœuvrent jusqu'à trois à la fois. Elle est très employée en Août et Septembre, à l'aube et au crépuscule, pour prendre Brochets, Perches, Truites et mème Blaufelchen et permet la capture de grosses pièces.

- Pour la pêche de l'Ombre, dans le Rhin, on utilise une ligne spéciale garnie de 10 à 12 hameçons dits "à plumes" ou d'appâts vivants.

Les lignes ci-dessus sont actionnées par l'homme. Celles qui suivent sont des lignes posées :

- La ligne de fond (Grundschnur ou Legschnur), ligne très longue munie de 200 à 500 hameçons et même davantage, maintenue sur le fond au moyen de pierres ou de plombs disposés de place en place. Elle permet la capture de Brochets, Perches, Truites, Lottes et Anguilles.

- La ligne flottante (Schwebangel ou Setzschnur), ligne très longue (souvent plusieurs centaines de mètres) maintenue en surface au moyen de flotteurs et ancrée par endroits. De place en place, se détachent verticalement des lignes secondaires munies d'hameçons appâtés dont le nombre peut varier, au total, de 200 à 300 . La profondeur des hameçons varie suivant la prise recherchée : en surface pour la Truite, de 3 à 4 mètres pour le Brochet, etc... La ligne flottante est utilisée surtout pendant l'hiver et permet la capture de grosses pièces.

On distingue :

\section{2. - Les filets.}

- Les filets traînants (Zuggarne ou Zugnetze);

- Les filets flottants (Schwebnetze);

- Les filets posés ou de fond (Stellnetze ou Grundnetze).

\section{A. - Filets trainants}

Il en existe de nombreux modèles qui, selon Scheffect, peuvent être rangés parmi les types suivants :

- Filets traînants sans sac. - Ce sont des filets à mailles lâches ou molles dans lesquelles le poisson se prend de lui-même. Le Lohmsegi ou Lohmgarn, autrefois utilisé dans le Lac Supérieur, avait 100 à 200 mètres de longueur, 15 à 20 mètres de haut; le Tiefensegi, semblable au précédent, était plus long et plus haut. fermes.

- Filets traînants avec sac. - Ces filets sont, au contraire, à mailles

Pour la pêche du Blaufelchen, dans le Haut Lac, on se sert du Klusgarn. C'est un filet constitué par deux ailes de 50 mètres chacune placées de part et d'autre d'un sac, formant entonnoir, de 8 mètres de 
longueur et dont les 4 derniers mètres (pointe) ont des mailles de 35 millimètres.

Cet engin agit sur 22 mètres de profondeur et est employé lorsque les fonds ont au moins 25 mètres. Les deux ailes se prolongent par des cordes de traction dont l'une, munie d'un flotteur placé à 45 mètres de l'aile, est mise à l'eau au début de l'opération, tandis que l'autre n'est jetée par-dessus bord qu'au fur et à mesure de l'avancement du bateau vers le flotteur. Lorsque celui-ci est atteint, on tire les deux ailes du filet. Le poisson est ainsi peu à peu dirigé vers le sac dans lequel il pénètre et qui est finalement ramené dans le bateau. La manœuvre est faite par deux hommes et dure un quart d'heure. Elle parmat la capture de 10 à 20 pièces en moyenne.

\section{Le Klusgarn est utilisé d'Avril à Octobre.}

Pour la pêche sur le mont et sur la beine, on emploie divers filets traînants (Haldenwatt, Landwatt, Gangfischsegi, Haselwatt, etc.) tirés d'un bateau ou de la rive et frôlant le sol. Leur longueur varie de 55 à 180 mètres, leur hauteur de 25 à 20 mìtres. Ces filets grands et lourds sont généralement maniés par quatre hommes.

Signalons encore qu'un privilège ancien accorde aux pêcheurs d'Ermatingen, le droit de procéder chaque année à deux captures près de Staad-Ermatingen, entre le 20 Novembre et le 25 Décembre, c'est-à-dire en période de frai. Ce droit provient d'un ancien fief du monastère de la Reichenau; il est mis à profit en commun par 18 pêcheurs d'Ermatingen qui utilisent un filet traînant long de 180 mètres avec sac de 20 mètres, à mailles de 35 millimètres (ailes) et 30 millimètres (sac). Le filet est tiré d'un grand bateau par 16 hommes, tandis que 2 observateurs surveillent le poisson et dirigent la manœuvre. L'engin permet de prendre jusqu'à 2.000 pièces à la fois et près de 50.000 en une saison.

\section{B. - Filets flottants}

Le type de ces filets est le : Schwebnetz, utilisé pour la pêche du Blaufelchen par les pêcheurs du Haut Lac; c'est leur seul engin avec le Klusgarn. Il est constitué par une nappe de filet de 6 à 25 mètres de hauteur, suspendue à l'aide de lignes munies de flotteurs. La nappe a 120 mètres de long et est formée d'èléments de $1 \mathrm{~m}$. 50 de largeur reliés l'un à l'autre. Les mailles sont d'au moins 40 millimètres. Le filet est simplement posé le soir et flotte librement. La pêche se produit sans l'action de l'homme durant la nuit. Le lendemain matin, on part à la recherche du filet, souvent dans le brouillard, pour le relever. La température et la pureté de l'eau indiquent à quelle profondeur on pourra espérer trouver le poisson, lui-même à la recherche du plancton durant l'été et l'automne. Au début de Décembre, en période de frai, on prend les poissons mûrs seulement en surface et il est interdit de tendre les filets au-delà d'une profondeur déterminée. Le clair de lune, qui éloigne le plancton et, peut-être, permet au poisson d'apercevoir le filet, est défavorable à la pêche. 
Pour le Gangfisch on utilise des Schwebnetze ancrés, à mailles de 30 millimètres seulement et maintenus à 15 mètres au moins au-dessous de la surface. Ils servent sur le mont en été, avant le frai du Gangfisch et ainsi ne prennent pas de jeunes Blaufelchen.

\section{C. - Filets posés ou filets de fond (Stellnelze ou Grundnetze).}

Leur partie inférieure touche le sol.

Les uns sont à panneau unique, les autres à plusieurs panneaux.

Les premiers, utilisés pour prendre le Brochet, la Perche, le Kilch, le Gangfisch, le Fera, le Barbeau, la Brême, la Carpe, etc., varient par la dimension de leurs mailles $(10$ à $20 \mathrm{~mm}$. pour l'Ablette, 70 à 80 pour la Brême en passant par 45 à 50 pour la Truite). La hauteur du filet varie : elle est de 5 mètres en général dans le Lac Inférieur. La réglementation fixe les périodes d'emploi des uns et des autres dans chacun des deux lacs. On dispose souvent le filet en spirale; il est maintenu vertical par des flotteurs et des plombs. Lors du frai du Fera, on le dispose en cercle pour traquer le poisson, ou bien l'on fait deux spirales droite et gauche maintenues par des piquets, le poisson étant préalablement guidé vers le milieu du filet par un panneau directeur placé perpendiculairement.

Les filets à plusieurs panneaux (Spiegelnetze ou filets miroir) analogues au tramail, comprennent trois parois unies l'une à l'autre par les mêmes brins de bordure, les deux parois extérieures étant très tendues et à mailles larges, l'intérieure à mailles étroites et lâches. Le poisson forme avec ces mailles lâches une poche qui l'emprisonne.

\section{3. - Les verveux (Reusen).}

Chacun connaît le principe des verveux. Ici, comme ailleurs, ces engins sont utilisés depuis un temps immémorial. Il en existe en filet, en fil métallique, ou faits avec des verges. Parfois, après une première chambre faisant suite à l'entonnoir de l'entrée, s'en trouve une ou plusieurs autres. Parfois aussi, il y a deux entonnoirs.

On utilise plutôt actuellement les verveux de $1 \mathrm{~m} .50$ de long et $0 \mathrm{~m}$. 50 de haut en filet ou en fil métallique.

Dans le Lac Inférieur, des verveux en osier de $0 \mathrm{~m}$. 40 de haut et $1 \mathrm{~m}$. 50 de long, servent à pêcher la Lotte; ils sont placés sur le fond en nombre variable atteignant parfois 50 , espacés de 5 mètres le long d'une corde.

Signalons enfin l'emploi près de Gottlieben et de Paradies, dans le Rhin, des "Fachenbehren ", constitués par des perches entre lesquelles 
sont tressées des branches de saule ou de hêtre formant parois de 15 à 20 mètres de long, inclinées d'environ $75^{\circ}$ l'une par rapport à l'autre. Entre chaque extrémité de ces parois, on laisse un espace libre de $0 \mathrm{~m} .50$ environ où se place l'entrée de verveux alternativement disposés face à l'amont et face à l'aval et dans lesquels pénètrent les poissons descendant ou remontant le courant. Ces engins permettent surtout la capture du Gangfisch en période de frai.

Dans le Lac Supérieur, le verveux ordinaire en filet est utilisé en période de frai dans les roseaux et près des embouchures des affluents.

Enfin, depuis 1925, on utilise aussi le verveux à ailes américain, appelé Trappnetz, devant lequel on dispose un panneau directeur de 70 mètres de long et 2 mètres de haut. Les ailes, fixées par des ancres, ont 6 mètres de long et 2 mètres de haut. Le panneau a des mailles de 40 millimètres au moins, le sac et les ailes des mailles de 30 millimètres. Ce trappnetz coûte cher, mais demande moins de travail, n'étant visité que tous les deux jours. Il est très utile pour la capture des poissons en temps de frai (Brochets en particulier et Sandres.)

\section{II. - PRODUCTION ET RENDEMENT}

Deux tableaux indiquent la production moyenne de la pêche pendant les années 1946 à 1949 et permettent de se faire une idée d'ensemble rapide et suffisamment exacte de cette production.

Nous tenons, toutefois, à mettre le lecteur en garde contre certains risques d'erreurs :

- Une moyenne sur quatre années n'est pas très étalée dans le temps, car les conditions naturelles au cours des années de référence peuvent avoir été exceptionnellement bonnes ou mauvaises.

- Par contre, les chiffres indiqués sont récents et, par conséquent, susceptibles de faire ressortir une modification en valeur absolue ou relative du peuplement du Lac.

- Aucune donnée précise n'existe sur le produit de la pêche sportive et celui-ci est certainement appréciable. Une mise au point, difficile mais nécessaire, serait à faire de ce chef.

- Pendant les années 1946 à 1949 les conditions naturelles paraissent avoir été moyennes dans l'ensemble, mais la production de la pêche allemande, du fait de difficultés nées de la guerre, a cependant été notablement inférieure, en 1946, 1947 et 1948, à ce qu'elle aurait dû être.

Ces réserves faites, nous croyons à la valeur des moyennes rapportées ici tant pour le Lac Supérieur que pour le Lac Inférieur. A côté du produit de la pêche, en kilogrammes, nous avons indiqué, pour la période 1946 à 1949 et pour l'année 1920, le pourcentage de chaque espèce de poisson dans la pêche totale. 


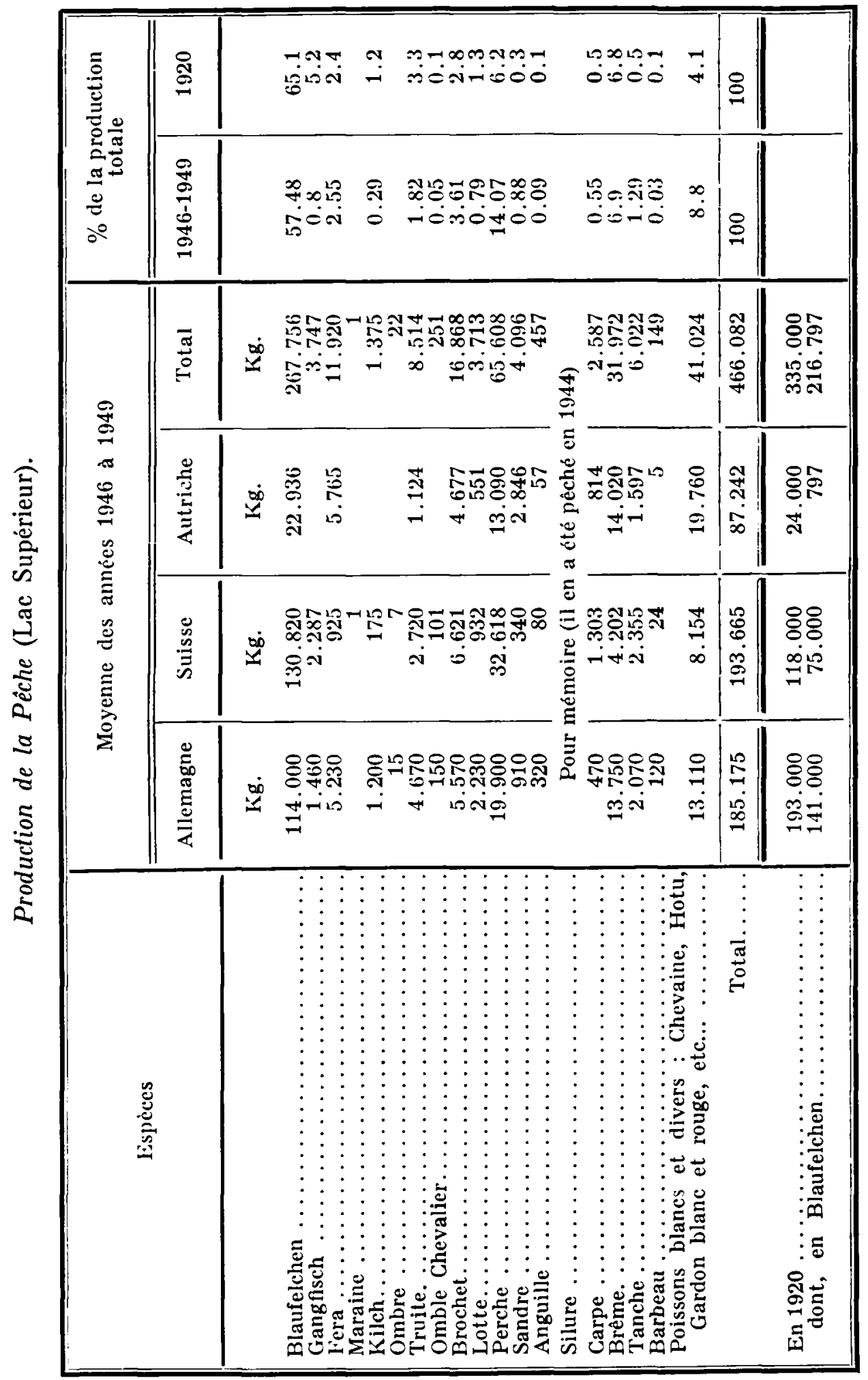


Production de la Pêche (Lac Inférieur).

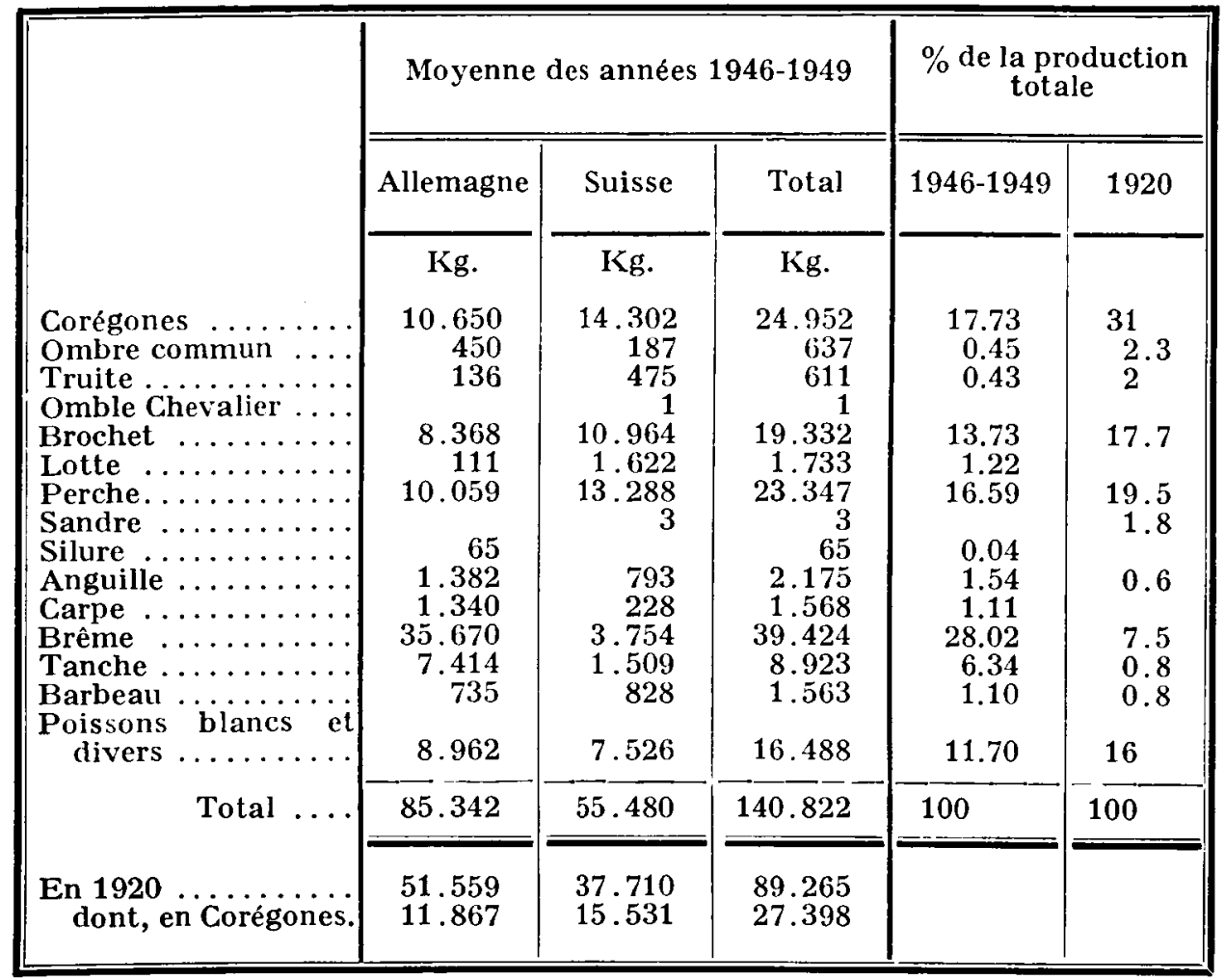

Ces deux tableaux appellent les commentaires ci-après :

\section{Lac Supérieur}

Le Blaufelchen paraît diminuer en pêche allemande et augmenter en pêche autrichienne.

En 1930, une moyenne établie sur dix années donnait, en effet, pour cette espèce de poisson, les chiffres approximatifs ci-après :

Pêche allemande. $\ldots \ldots \ldots \ldots \ldots \ldots \ldots \ldots \ldots, \quad 150.000 \mathrm{kgs}$.
Pêche suisse. $\ldots \ldots \ldots \ldots \ldots \ldots \ldots \ldots \ldots \ldots$
Pèche autrichienne. $\ldots \ldots \ldots \ldots \ldots \ldots \ldots \ldots$

En fait, la diminution du Blaufelchen en pêche allemande est uniquement une conséquence de la guerre, tandis que l'accroissement des captures par les pêcheurs autrichiens résulte de la Convention Internationale de 1915, autorisant sous certaines conditions la pêche du Haut Lac par les pêcheurs de tous les États riverains.

Dans l'ensemble, le Blaufelchen est en augmentation et la réglementation de sa pêche, étant donnée son importance économique, fait l'objet 
'de soins particulièrement attentifs de la part de la Commission Internationale.

Le Gangfisch, à l'inverse de ce que l'on constatait il y a vingt ans, paraît diminuer. Cette espèce, dont la vie est mal connue, serait concurrencée, croit-on, par d'autres espèces, telles que la Perche et le Brochet, ou affectée par la pollution de ses zones d'habitat ou encore par l'accroissement de la navigation et le trouble qui en résulte. Le Gangfisch étant la seule espèce atteinte, le premier motif paraît le plus plausible et le plus naturel.

Le Fera, poisson intéressant par sa croissance rapide, augmente dans l'ensemble, surtout en Allemagne et en Autriche. En Suisse, il a un peu diminué.

Le Kilch semble diminuer.

Les Truites se maintiennent difficilement, sauf vers l'Autriche.

L'Omble Chevalier semble diminuer.

Le Brochef augmente partout. Il faut rappeler, à son propos, qu'il est l'objet, dans les deux lacs, d'une pisciculture artificielle intensive et que, de plus, certaines zones ont été mises en réserves (réserves spéciales de places à roseaux), mais ces réserves sont fortement concurrencées par les constructions nombreuses faites sur les rives du Lac (murs et travaux divers de maçonnerie, aménagements de plages.)

La Perche augmente partout notablement, sans toutefois se multiplier au point de concurrencer par trop les Corégones, sauf peut-être le Gangfisch.

La Lotte est stationnaire, sauf en Autriche où elle serait en régression.

Le Sandre augmente partout, mais l'introduction régulière d'alevins reste encore indispensable, à notre avis.

L'Anguille se maintient.

La Carpe tend à augmenter, mais c'est surtout la Tanche qui s'est multipliée, notamment en Allemagne et en Suisse, sa pèche dépassant six tonnes pour tout le Lac.

Quant à la Brême, elle augmente aussi, sauf en Suisse.

Les divers (blanchaille) ont tendance à augmenter partout.

\section{LAC INFÉRIEUR}

Les divers Corégones sont en nette diminution, malgré un élevage intensif. Cependant, en 1937, le $D^{r}$ Косн avait relevé une augmentation, qui ne s'est plus maintenue.

L'Ombre commun, déjà en forte régression en 1937, continue à diminuer; il serait urgent de le protéger par une réglementation appropriée. La Truite diminue également très nettement, mais sa pèche est beaucoup plus irrégulière et il faut, de plus, tenir compte du fait que son alevinage artificiel vient de la Haute-Vallée du Rhin (Grisons et Vorarlberg).

En tout cas, ces trois espèces nobles ont grand peine à se maintenir et on en peut voir la cause principale dans la pollution du Lac. On peut en dire de même du Sandre, qui tend à disparaître. 
Le Brochet, la Perche, les poissons blancs sont en meilleure situation, bien qu'en diminution par rapport à 1937 .

L'augmentation de la Brême, déjà signalée en 1937 par le $\mathrm{D}^{\mathrm{r}} \mathrm{Koch,}$ s'est encore accrue depuis. Ne faut-il pas voir là aussi l'effet de la pollution du Lac? Moins exigeante en oxygène, la Brême se développe au détriment des espèces nobles.

La Tanche se développe également beaucoup, mais il faut noter qu'elle fait l'objet, à bon droit, d'un repeuplement régulier par déversements d'alevins effectués notamment par l'Association de pêche professionnelle badoise de la Reichenau.

Signalons, enfin, une augmentation de la Lotfe, de l'Anguille et du Barbeau (celui-ci limité au Rhin et sans grande importance) et de la Carpe (sans grande importance non plus).

En Allemagne, on classe habituellement les grands lacs en cinq classes, suivant leur production à l'hectare :

$\begin{array}{llll}1 \text { re } & \text { classe } \ldots \ldots & 80 \mathrm{kgs} \text { et au-dessus } \ldots \ldots \ldots & \text { Très bons. } \\ 2^{\mathrm{e}} & \text { classe } \ldots \ldots & 40 \text { à } 80 \mathrm{kgs} . \ldots \ldots \ldots \ldots \ldots & \text { Bons. } \\ 3^{\mathrm{e}} & \text { classe } \ldots \ldots & 20 \text { à } 40 \mathrm{kgs} . \ldots \ldots \ldots \ldots & \text { Moyens. } \\ 4^{\mathrm{e}} & \text { classe } \ldots \ldots & 10 \text { à } 20 \mathrm{kgs} . \ldots \ldots \ldots \ldots & \text { Médiocres. } \\ 5^{\mathrm{e}} & \text { classe } \ldots \ldots & \text { Au-dessous de } 10 \mathrm{kgs} . \ldots \ldots & \text { Mauvais. }\end{array}$

Pour calculer ce rendement pour la zone littorale, d'une part, et pour la zone pélagique, d'autre part, nous admettrons tout d'abord les données de Scheffelt et de Schweizer sur l'importance relative de la beine et du large.

Dans le Lac Supérieur, dont la superficie est d'environ 475 kilomètres carrés, on admet que la beine représente approximativement un dixième de la superficie totale et qu'elle couvre donc 4.750 hectares, le Haut Lac occupant le surplus, soit 42.750 hectares.

Il y a trente ans, on évaluait le produit de la pêche côtière aux trois dixièmes de la pêche totale. Nous admettrons cette même proportion pour la période 1946-1949 et nous obtiendrons ainsi les résultats ci-après :

Rendement par hectare et par an.

\begin{tabular}{|c|c|c|}
\hline Zone & En 1920 & $1946-1949$ \\
\hline & Kgs. & Kgs. \\
\hline Beine & 21 & 29,5 \\
\hline Haut Lac. & 5,5 & 7,6 \\
\hline Lac entier. & 7 & 9,8 \\
\hline
\end{tabular}


Ce tableau fait ressortir une augmentation sensible du rendement, la zone "Beine " tendant vers la deuxième classe, tandis que le Lac, pris dans son ensemble, aborde la quatrième.

Dans le Lac Inférieur, dont la surface totale est de 63 kilomètres carrés, il est admis que la beine atteint presque les $4 / 10^{\mathrm{e}}$ de la surface du lac, soit 2.520 hectares, ce qui explique d'ailleurs le rendement très supérieur de ce Lac, très eutrophié, menacé toutefois très fortement par sa pollution progressive. Adoptant un rendement "beine " de 24 kilogrammes en 1920, ce qui semble un peu au-dessous de la vérité, on obtient le tableau suivant :

Rendement par hectare et par an.

\begin{tabular}{|c|c|c|}
\hline Zone envisagée & En 1920 & En 1946-1949 \\
\hline & Kgs. & Kgs. \\
Beine $\ldots \ldots \ldots \ldots \ldots \ldots \ldots \ldots \ldots \ldots \ldots \ldots \ldots$ & 24 & 37,7 \\
Zone pélagique $\ldots \ldots \ldots \ldots \ldots \ldots \ldots$ & 7,5 & 12,10 \\
Lac entier $\ldots \ldots \ldots \ldots \ldots \ldots$ & 14,1 & 22,2 \\
\hline
\end{tabular}

Ici également la production est en augmentation. Dans son ensemble le Lac Inférieur tend vers la deuxième classe, mais il est regrettable que, par ailleurs, il présente des signes évidents de pollution. La nature de son peuplement piscicole l'indique déjà.

\section{CONCLUSION}

Le travail que nous avons entrepris sur le Bodensee et que le Bulletin Français de Pisciculture a reproduit dans ses numéros 159 (Oct.-Déc. 1950: Aperçu géographique, Hydrographie, Climat, Caractères physiques et chimiques, Hydrobiologie), 160 (Janv.-Mars 1951 : Faune piscicole), 161 (Avril-Juin 1951 : Législation, Réglementation et Exercice de la pêche), 163 (Oct.-Déc. 1951 : Equipement piscicole) et 165 (Janv.-Mars 1952 : Exploitation piscicole), aurait pu être beaucoup plus développé. Nous avons dû choisir dans la documentation recueillie au cours de la mission dont nous avons été chargé à Constance de 1945 à 1950, les éléments qui nous ont paru essentiels pour donner de ce magnifique fonds d'eau qu'est le Bodensee, une image concise mais cependant aussi complète et exacte que possible.

Nous espérons l'avoir fait judicieusement.

Nous voudrions avoir montré également l'intérêt que nous avons eu à pouvoir suivre en terre étrangère les réalisations des savants et des 
techniciens d'Allemagne, d'Autriche et de Suisse et les heureuses habitudes de collaboration, qu'ils ont prises depuis longtemps pour y aboutir.

La pêche du Lac de Constance est placée en de bonnes mains et il est probable que les études nombreuses et approfondies dont elle est l'objet, assureront sa conservation, sinon son développement, tout en apportant une fructueuse contribution à la limnologie européenne en général. Il apparaît même, d'après notre exposé, que le Lac Supérieur conserve des eaux de qualité et améliore son rendement; par contre, la qualité du Lac Inférieur diminue nettement. La surveillance de la qualité des eaux des deux lacs est, en premier lieu, l'affaire des Instituts de Recherches ; ils s'y emploient avec beaucoup de dynamisme. Leur travail est le premier qu'il convient d'assurer pour éviter, dans les conditions de vie des poissons, un déséquilibre soudain qui placerait brusquement les techniciens en face de problèmes presque insolubles posés par le recul continu de la Nature devant l'Homme. Le "Progrès ", bon en soi, exige une saine discipline et des règles de prudence, que seuls des hommes prévoyants peuvent proposer en temps voulu.

Il nous reste, pour terminer, un devoir bien agréable à remplir. C'est celui de remercier très vivement tous ceux qui ont bien voulu nous aider de leurs connaissances et de leurs travaux : $M$. le Commissaire suisse Mathey-Doret, Inspecteur fédéral de la pêche, haut fonctionnaire si affable et si compétent, M. le Professeur Auerbach, MM. les Docteurs. Nümann, Muckle, Grim, M. l'Oberregierungsrat Docteur Koch, M. Beck, M. le Docteur Bernhardt, Commissaire adjoint pour l'Autriche; MM. les Docteurs Schuly et Schiess, le Maître de Pêche Oskar Косн, dont la famille travaille depuis près de trois siècles à la pêche du Lac Inférieur, et, pour n'oublier personne, tous les Services scientifiques, techniques et de surveillance du Bodensee. 